Boise State University

ScholarWorks

5-1-2011

Simultaneous Measurement of Normal and Friction Forces Using a Cantilever-Based Optical Interfacial Force Microscope

Byung I. Kim

Boise State University

Jeremy R. Bonander

Boise State University

Jared A. Rasmussen

Boise State University 


\title{
Simultaneous Measurement of Normal and Friction Forces Using a Cantilever-Based Optical Interfacial Force Microscope
}

\author{
Byung I. Kim, Jeremy R. Bonander, and Jared A. Rasmussen \\ Boise State University
}

\begin{abstract}
We measured normal and friction forces simultaneously using a recently developed cantileverbased optical interfacial force microscope (COIFM) technique for studies of interfacial structures and mechanical properties of nanoscale materials. We derived how the forces can be incorporated into the detection signal using the classical Euler equation for beams. A lateral modulation with the amplitude of one nanometers was applied to create the friction forces between tip and sample. We demonstrated its capability by measuring normal and friction forces of interfacial water at the molecular scale over all distance ranges.
\end{abstract}

Keywords: COIFM, cantilever-based optical interfacial force microscope, force-distance curve, friction force, interfacial force microscopy

\section{Introduction}

Intermolecular friction forces play a fundamental role in many biological processes such as transport along cytoskeletal filaments ${ }^{1}$ or inside human and animal joints ${ }^{2}$. Friction is also of great importance in micromechanical systems where water is trapped between two surfaces ${ }^{3}$. The trapped water has a critical effect on the performance of the systems through interfacial tribological properties ${ }^{4}$.

Atomic force microscopy (AFM) has been successfully applied to these intermolecular friction studies ${ }^{5-11}$. The previous technique of measuring friction employed a lateral modulation of the sample relative to the cantilever as a means to measure normal force and friction force at the same time $e^{7-11,13}$. However, the technique can only be applied to the measurement of friction while the tip is touching because of an intrinsic mechanical instability of the tip-sample assembly called the snap-to-contact problem. As an approach to overcome these limitations, friction forces have been measured using interfacial force microscopy (IFM) ${ }^{12}$, where the force feedback makes it possible to study friction in the attractive regime ${ }^{10-11,13}$. Burns et al. applied IFM to measure friction force and normal force simultaneously to study the molecular nature of friction to investigate the intermolecular friction along with normal forces in the attractive regime ${ }^{13}$. However, its use of a larger tip and its limited force resolution determined by the electrical detection technique do not allow for broad use in friction force measurements, especially at the single molecular level.

We employed the unique and recently developed scanning probe technique called cantilever-based optical interfacial force microscope (COIFM) ${ }^{14}$, which uses force feedback to avoid the snap-to-contact problem associated with ordinary AFM measurements. The COIFM with lateral modulation allows for simultaneous measurement of normal and friction forces in the attractive regime as well as in the repulsive regime by utilizing the force feedback capability of the instrument ${ }^{14}$. Like the conventional IFM, ${ }^{10-12}$ the COIFM uses a voltage-activated force feedback by employing a commercially available "dimension micro-actuated silicon probe" (DMASP) cantilever. ${ }^{15-18}$. This cantilver has a small radius $(10 \mathrm{~nm})$ that enables one to study water structures at the single molecular level. Due to the optical detection scheme, the force resolution is improved by two orders of magnitude over the existing IFM with electrical detection method ${ }^{12}$. It also demonstrated the capability of this COIFM as a second generation of IFM by revealing the hidden structures of the interfacial water between two silica surfaces ${ }^{14}$. Although there have been many friction measurements using scanning probe techniques, there are few explicit relations between the detection signal, normal, and frictional forces. In this paper, we derived the relationship between the detection signal, $\mathrm{V}_{\mathrm{A}-\mathrm{B}}$, an external normal force $\left(F_{z}\right)$ and an external friction force $\left(F_{x}\right.$, and using the classical Euler equations for beams ${ }^{19}$. Based on this relationship, we calibrated the conversion factors between detection signals and forces. Then we demonstrated its capabilities through measurement of normal and friction forces due to interfacial water using the COIFM. 
This is an author-produced, peer-reviewed version of this article. The final, definitive version of this document can be found online at Review of Scientific Instruments, published by American Institute of Physics. Copyright restrictions may apply. DOI: $10.1063 / 1.3593106$

\section{Theoretical Background}

\section{a. Coupling of Normal and Friction forces through Cantilever Displacement}

Herein we describe the theory behind the COIFM with lateral modulation and demonstrate its capabilities in measuring normal and friction forces at the single molecular level due to interfacial water. Figure 1 illustrates an optical beam displacement detection scheme in the AFM head that was used to transmit the interaction forces between the tip and the surface into an electrical signal ${ }^{20}$. The tip of the cantilever experiences the forces $F_{\mathrm{x} \text { and }} F_{z}$ during force measurements by the sample surface. A general Euler equation ${ }^{19}$ is given for the vertical displacement of the cantilever $\left(z_{z}\right)$ produced by the normal force $\left(F_{z}\right)$ acting at a point $\mathrm{x}=L_{\text {cant }}$ as follows ${ }^{21,22}$,

$$
F_{z}\left(L_{\text {cant }}-x\right)=E I \frac{d^{2} z_{z}}{d x^{2}}
$$

where $L_{\text {cant }}$ is the length of the cantilever, $E$ is the Young's Modulus and $z_{z}$ is the vertical displacement caused by $F_{z}$. The area moment of inertia $(I)$ for a rectangular bar is given by,

$$
I=\frac{t^{3} w}{12}
$$

where $t$ is the bar thickness, and $w$ is the width of the bar. The solution to the above equation with the boundary

$$
\begin{gathered}
\text { condition }\left.z_{z}\right|_{z=0}=0,\left.\frac{d z_{z}}{d x}\right|_{z=0}=0 \text {, is as follows, } \\
z_{z}=\frac{3 F_{z}}{2 k_{z}}\left(\frac{x}{L_{\text {cant }}}\right)^{2}\left(1-\frac{x}{3 L_{\text {cant }}}\right)
\end{gathered}
$$

where the spring constant $\left(k_{z}\right)$ is defined as the following ${ }^{23-25}$

$$
k_{z}=\frac{E t^{3} w}{4 L_{\text {cant }}^{3}} .
$$

In addition to the vertical force $F_{z}$, a friction force $\left(F_{x}\right)$ along the major axis of the cantilever (see Fig. 1(a)) also contributes to the vertical displacement of the cantilever by the following Euler equation.

$$
F_{x} L_{\text {tip }}=E I \frac{d^{2} z_{x}}{d x^{2}}
$$

where $L_{t i p}$ is the length of the cantilever tip. The vertical displacement produced by $F_{x}\left(z_{x}\right)$ can then be found in the following equation. 


$$
z_{x}=\frac{3 F_{x}}{2 k_{z}} \cdot \frac{L_{\text {tip }}}{L_{\text {cant }}}\left(\frac{x}{L_{\text {cant }}}\right)^{2}
$$

The total displacement $\left(z_{c}\right)$, the sum of both $z_{z}$ and $z_{x}$, is given by the following equation.

$$
z_{c}=\frac{3}{2 k_{z}}\left(\frac{x}{L_{\text {cant }}}\right)^{2}\left[\frac{L_{\text {tip }}}{L_{\text {cant }}} F_{x}+\left(1-\frac{x}{3 L_{\text {cant }}}\right) F_{z}\right]
$$

The bending motion of the cantilever due to the tip-sample interactions is detected by measuring the voltage difference $\left(\mathrm{V}_{\mathrm{A}-\mathrm{B}}\right)$ between two photodiodes, namely $\mathrm{A}$ and $\mathrm{B}$, as shown in Figure 1. The difference in voltage is proportional to the slope of the cantilever (according to the law of reflection) at the point ( $x=L_{\text {cant }}$ ) where the beam is reflected as follows ${ }^{26}$,

$$
V_{A-B}=\alpha \vartheta_{C}
$$

where $\alpha$ is a proportional constant.

The total slope ( $\vartheta_{c}$ ) at $x=L_{\text {cant }}$ is the derivative of total displacement of the cantilever (as given in equation (7)) with respect to $\mathrm{z}$ in the following way.

$\vartheta_{c}=\left.\frac{d z_{c}}{d x}\right|_{x=L_{\text {cant }}}=\frac{3}{2 k_{z} L_{\text {cant }}}\left(F_{z}+2 \frac{L_{\text {tip }}}{L_{\text {cant }}} F_{x}\right)$.

The above relation is consistent with the work done by $\mathrm{Sader}^{24}$. Then the detection signal $\mathrm{V}_{\mathrm{A}-\mathrm{B}}$ is related to the two forces as follows:

$$
V_{A-B}=\frac{3 \alpha}{2 k_{z} L_{\text {cant }}}\left(F_{z}+\frac{2 L_{\text {tip }}}{L_{\text {cant }}} F_{x}\right)
$$

The above relation provides an opportunity to measure the normal force $F_{\mathrm{z}}$ and the friction force $F_{x}$ simultaneously through the measurement of the optical beam displacement signal, $\mathrm{V}_{\mathrm{A}-\mathrm{B}}$. However, the inability of the current AFM system to control the cantilever displacement causes limitations in measuring forces using Eq. (10) over all distance ranges due to the snap-to-contact problem.

\section{b. Voltage Activated Force Feedback}

We apply the concept of the COIFM technique to the simultaneous measurement of normal and friction forces on approach to overcome the limitations of the AFM, using a commercially available DMASP cantilever as a detector and an actuator. Here we apply this concept to the case where friction forces $\left(F_{x}\right)$ and normal force $\left(F_{z}\right)$ exist together by making displacement zero while measuring the normal force $F_{z}$ through the voltage activated force feedback ${ }^{14}$. When in feedback, the detector signal $\left(\mathrm{V}_{\mathrm{A}-\mathrm{B}}\right)$ in Figure $\mathbf{1}$ is maintained at zero by generating a feedback force $F_{\text {feedback }}$ as follows. 


$$
V_{A-B}(t)=\frac{3 \alpha}{2 k_{z} L_{c a n t}}\left(F_{z}+2 \frac{L_{\text {tip }}}{L_{\text {cant }}} F_{x}-F_{\text {feedback }}\right)=0
$$

In the voltage activated force feedback, a voltage $\mathrm{V}_{\mathrm{ZnO}}$ is applied to the $\mathrm{ZnO}$ stack of the DMASP cantilever. Because $\mathrm{V}_{\mathrm{ZnO}}$ is linearly proportional to $\mathrm{V}_{\mathrm{A}-\mathrm{B}}$ with a proportional constant $\beta$ as given,

$$
V_{A-B}=\beta \cdot V_{Z n O}
$$

the feedback condition in equation (11) can be solved in terms of $\mathrm{V}_{\mathrm{ZnO}}$ as follows.

$$
V_{Z n O}=\frac{3 \alpha}{2 \beta k_{z} L_{\text {cant }}}\left(F_{z}+\frac{2 L_{\text {tip }}}{L_{\text {cant }}} F_{x}\right)
$$

This equation suggests that the ability to obtain normal and friction forces while overcoming the snap-to-contact problem will make feedback measurements much more advantageous than non-feedback measurements. Equation (13) suggests, instead of the $\mathrm{V}_{\mathrm{A}-\mathrm{B}}$ signal, the feedback signal $\mathrm{V}_{\mathrm{ZnO}}$ is used in measuring normal and friction forces.

\section{Experimental}

We used a commercially available AFM system, which was originally designed for general purpose use of AFM (Autoprobe LS, Park Scientific Instruments, Sunnyvale, CA). For our experiment, we used a (DMASP, Bruker Corporation, Santa Barbara, CA), which has a built-in ZnO self-actuator but can still be used as a regular AFM tip. The DMASP cantilever acts as not only a detector, but also an actuator as well due to the ZnO stack. This probe is made of 1-10 $\Omega \mathrm{cm}$ Phosphorus doped Si, with a nominal spring constant $\left(k_{z}\right)$ and resonance frequency known to be $3 \mathrm{~N} / \mathrm{m}$ and $50 \mathrm{kHz}$ respectively ${ }^{27}$. The dimensions were measured to be $L_{\text {cant }}=485 \mu \mathrm{m}, L_{\text {tip }}=20 \mu \mathrm{m}$, which is in agreement with other previous measurements ${ }^{28,29} \cdot L_{\text {cant }}$ is the length of the portion of the cantilever between the base of the cantilever and the tip. Before experimentation, the laser beam was aligned on the backside of the cantilever and A-B was adjusted to make the laser incident zero by reflecting in the middle of the photo-diode. The tip then approached the sample until touching, using the stepping motor of the piezotube. The wavelength of the laser light for the optical detection is $670 \mathrm{~nm}$ and the position sensitive detector is a bi-cell silicon photo-diode. The feedback loop was developed using a RHK SPM 1000 controller (RHK Technology Inc., Troy, MI). The feedback control parameters such as time constant and gain can be manually adjusted for the optimal feedback condition. All measurements were taken on a freshly cleaned silica wafer, Si (100) (SPI supplies, West Chester, PA) in an ambient condition with a relative humidity of 55\%, which was monitored using a thermo-hygro recorder (Control3, Friendswood, TX). The top of the surface is expected to becovered with natural oxide in air, thereby forming the silica, the most abundant material in the Earth's crust ${ }^{30}$. The wafer was attached to a 15 mm steel disk using double stick tape and then mounted on a magnetic sample stage on top of the piezotube. To remove all organic contaminates the silica was cleaned using a piranha solution made from a 3:1 concentrated $\mathrm{H}_{2} \mathrm{SO}_{4} / 30 \% \mathrm{H}_{2} \mathrm{O}_{2}$ (Pharmco and Fischer Scientific respectively). It was then sonicated in acetone for 5 minutes, then in ethanol for 5 minutes, rinsed with DI water, and then dried with a dry $\mathrm{N}_{2}$ flow. Tips were cleaned using a UV sterilizer (Bioforce Nanosciences Inc., Ames, IA) to remove the residual hydrocarbon molecules.

The tip-sample distance was controlled by moving the piezotube in the z-direction using the high-voltage signal controller. The zero force was set as the $\mathrm{V}_{\mathrm{A}-\mathrm{B}}$ value at large separations between the two surfaces before measurement. The tip speed was chosen as $10 \mathrm{~nm} / \mathrm{sec}$, controlled by the built-in digital-to-analog converter of the RHK controller in conjunction with the high voltage amplifier. The lateral movement was achieved by dithering the sample in the long axis direction of the cantilever using the piezo tube with an oscillatory signal of about $1 \mathrm{~nm}$ amplitude at the frequency of $100 \mathrm{~Hz}^{9-11}$. A Hewlett-Packard function generator (model 33120A) was used to generate the oscillatory signal. The piezotube sensitivities in the $\mathrm{x}$ and $\mathrm{y}$-direction are calibrated to be $6.25 \mathrm{~nm} / \mathrm{V}$ and the sensitivity in the z-direction is $3.65 \mathrm{~nm} / \mathrm{V}$. 


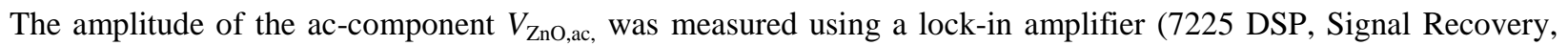
Oak Ridge, TN) with a time constant and sensitivity, $100 \mathrm{~ms}$ and $100 \mathrm{mV}$ (or voltage gain of 100), respectively. $\mathrm{V}_{\mathrm{A} \text { - }}$ B, $\mathrm{V}_{\mathrm{ZnO} \text {,dc }}$ and the lock-in output $\mathrm{V}_{\mathrm{ZnO} \text {,ac }}$ were recorded using the analog-to-digital converter of the RHK controller system. They were converted into forces using the conversion factors found in the results section. The converted force scales are displayed on the right axis of each panel and voltage units on the left axis. All data processing and analysis were performed with Kaleidagraph (Synergy Software, Reading, PA) after raw data acquisition.

\section{Results and Discussion}

\section{a. Normal and Friction force Calibration}

For qualitative understanding, the signal $\mathrm{V}_{\mathrm{A}-\mathrm{B}}$ should be converted into force using the relation between the signal and the two forces in equation (10). Equation (10) suggests how to calibrate the conversion factors from the $\mathrm{V}_{\mathrm{A}-\mathrm{B}}$ signal to both forces experimentally. To do this we need to find the proportional constant $\alpha$ between $\mathrm{V}_{\mathrm{A}-\mathrm{B}}$ and $\vartheta_{c}$. Combining the equation (8) with equation (10), the detection signal, when the tip is in contact with the substrate can be expressed as

$$
V_{A-B}=\frac{3 \alpha}{2 L_{c a n t}} z_{c}
$$

where $z_{c}$ is the cantilever displacement along z-axis.

The above equation suggests that $\alpha$ can be found by measuring the $\mathrm{V}_{\mathrm{A}-\mathrm{B}}$ signal as a function of $z_{c}$. The cantilever displacement can be changed systematically by contacting the cantilever to the sample surface (assuming that the indentation between the tip and the surface is negligible). The slope of the line $\left(\frac{3 \alpha}{2 L_{\text {cant }}}\right)$ is found to be 20.4 $\mathrm{mV} / \mathrm{nm}$ from the relationship between $\mathrm{V}_{\mathrm{A}-\mathrm{B}}$ and $z_{c}$ shown in Figure 2(a). From Figure 2(a), $\alpha$ is found to be $6.60 \times 10^{3} \mathrm{~V} / \mathrm{rad}$ with $\mathrm{L}_{\text {cant }}=485 \mu \mathrm{m}$. The normal force conversion factor from $\mathrm{V}_{\mathrm{A}-\mathrm{B}}$ into $\mathrm{F}_{\text {int }}$ from equation

$$
\text { (29), } \frac{2 k_{z} L_{c a n t}}{3 \alpha} \text {, is } 147 \mathrm{nN} / \mathrm{V} \text {. }
$$

Figure 2(b) shows the detection signal $\mathrm{V}_{\mathrm{A}-\mathrm{B}}$ measured as a function of the voltage applied to the $\mathrm{ZnO}$ stack from -10 $\mathrm{V}$ to $10 \mathrm{~V}$. The constant $\beta$ is found to be $34.07 \mathrm{mV} / \mathrm{V}$ through the linear fitting of the curve in Figure 2(b). Using the obtained $\alpha$ value along with the measured $\beta$ value, the calculated conversion factor for normal force while the system is in feedback $\left(\frac{2 \beta k_{z} L_{\text {cant }}}{3 \alpha}\right)$ is $5.00 \mathrm{nN} / \mathrm{V}$. The conversion factor for friction forces, $\frac{\beta k_{z} L_{\text {cant }}^{2}}{3 \alpha L_{\text {tip }}}$ found in equation (28) is calculated to be $60.62 \mathrm{nN} / \mathrm{V}$. This lateral conversion factor is larger than the normal force conversion factor by $\frac{L_{\text {cant }}}{2 L_{\text {tip }}}$ which is found to be 12.13 .

\section{b. Force-Distance Measurements}

We measured both normal and friction forces in the water junction between the probe and the surface with the COIFM with lateral modulation. Figure 3(a), 3(b) and 3(c) show the measured $\mathrm{V}_{\mathrm{A}-\mathrm{B}}, \mathrm{V}_{\mathrm{ZnO}, \mathrm{ac}}$, and $\mathrm{V}_{\mathrm{ZnO} \text {,dc }}$ data, respectively, as a function of piezo-displacement. The data clearly demonstrate that the force feedback allows for the COIFM to measure the normal force of water between the tip and the sample for all distance regimes, overcoming the snap-to-contact effect associated with the conventional AFM method. The voltage units were converted into 
This is an author-produced, peer-reviewed version of this article. The final, definitive version of this document can be found online at Review of Scientific Instruments, published by American Institute of Physics. Copyright restrictions may apply. DOI: $10.1063 / 1.3593106$

force units using the conversion factors found above, as shown on the right axis of each panel. The zero distance was defined as the point where the friction force increases sharply as marked in Figure 3(b). As the tip approaches, both normal and friction forces remain at zero until interaction with interfacial water occurs at around 12 nm away from the substrate. Surprisingly, the data show oscillatory patterns in both normal force and friction force. The $\mathrm{V}_{\mathrm{A}-\mathrm{B}}$ signal also displays a periodic change with the tip-sample distance, as shown in an enlarged inset in Fig. 3(a). These periodic features are consistent with the earlier AFM-based observation of stepwise change of the force gradient related with the thin water bridge in an ambient environment ${ }^{31}$.

The periodicity is found to be $0.227 \pm 0.056 \mathrm{~nm}$ for valley-valley distance analysis and $0.223 \pm 0.055 \mathrm{~nm}$ for peakpeak distance analysis. This periodicity matches the diameter of water, which is consistent with Antognozzi et. al, who found the periodicity of water layers to be $0.24-0.29 \mathrm{~nm}$ even for distilled water deposited on a mica sample surface using a near field scanning optical microscope (NSOM) ${ }^{32}$. This result is also in agreement with other earlier studies using AFM at the liquid-solid interface between a hydrophilic surface (e.g mica.) and bulk water ${ }^{33-38}$. The interfacial water confined between two surfaces forms water layers with periodicity of one water diameter 0.22 $\mathrm{nm}^{33}, 0.23 \pm 0.003 \mathrm{~nm}^{34}, 0.25 \pm 0.05 \mathrm{~nm}^{35}, 0.23 \pm 0.03 \mathrm{~nm}^{36}, 0.29 \pm 0.006 \mathrm{~nm}^{37}$, and 0.22-0.29 $\mathrm{nm}^{38}$. This can be understood that the ordering of confined water molecules leads to oscillatory solvation forces, which are reflections of the geometric packing experienced by the molecules due to the imposing surfaces, with the period of oscillation roughly equal to the molecular diameter of water. The oscillations occur in the molecular force due to the transition between solid (ordering) and liquid (disordering), depending on the commensuration and incommensuration between the spacing and the molecular diameter ${ }^{39}$. These preliminary data on the interfacial water suggest that the COIFM is capable of providing unprecedented information on the structural and mechanical properties of molecules.

\section{Acknowledgment}

Funding for this work was made possible by NSF DBI-0852886, NSF EPSCOR Startup Augmentation Funding, and the Research Corporation Single-Investigator (Cottrell College Science Award No. CC7041/7162). 
This is an author-produced, peer-reviewed version of this article. The final, definitive version of this document can be found online at Review of Scientific Instruments, published by American Institute of Physics. Copyright restrictions may apply.

\section{References}

[1] M.J.I. Mueller, S. Klumpp, and R. Lipowsky, Biophys. Journ. 98, 2610 (2010).

[2] M. Flannery, S. Flanagan, E. Jones, and C. Birkinshaw, Wear 5-6, 325 (2010).

[3] K. Komvopoulos, J. of Adh. Sci. \& Tech. 17, 477 (2003).

[4] M.P. de Boer and T.M. Mayer, MRS Bull. 26, 302 (2001).

[5] B.I. Kim, S. Lee, R.L. Guenard, L.C. Ferneandez-Torres, S.S. Perry, P. Frantz, and S.V.

Didziulis, Surf. Sci. 481, 185 (2001).

[6] L.C. Fernandez-Torres, B.I. Kim, and S.S. Perry, Tribol. Lett. 15, 43 (2003).

[7] R.W. Carpick, D.F. Ogletree, and M. Salmeron, Appl. Phys. Lett. 70, 1548 (1997).

[8] J. Colchero, M. Luna, and A.M. Baró, Appl. Phys. Lett. 68, 2896 (1996).

[9] T. Goddenhenrich, S. Muller, and C. Heiden, Rev. Sci. Instrum. 65, 2870 (1994).

[10] R.C. Major, J.E. Houston, M.J. McGrath, J.I. Siepmann, and X.Y. Zhu, Phys. Rev. Lett. 96, 177803 (2006)

[11] M.P. Goertz, J.E. Houston, and X.Y. Zhu, Langmuir 23, 5491 (2007)

[12] S.A. Joyce and J.E. Houston, Rev. Sci. Instrum. 62, 710 (1991).

[13] A.R. Burns, J.E. Houston, R.W. Carpick, and T.A. Michalske, Phys. Rev. Lett. 82, 1181 (1999).

[14] J.R. Bonander and B.I. Kim, Appl. Phys. Lett. 92, 103124 (2008).

[15] E. Meyer, H. Heinzelmann, P. Grütter, T.H. Jung, T. Weisskopf, H.R. Hidber, R. Lapka, H. Rudin, and H.J. Güntherodt, J. Microsc.-Oxford 152, 269 (1988).

[16] N.A. Burnham and R.J. Colton,. J. Vac. Sci. Technol. A. 7, 2906 (1989).

[17] K.B. Lodge, Adv. Coll. Interface Sci. 19, 27 (1983).

[18] J.P. Aimé, Z. Elkaakour, C. Odin, T. Bouhacina, D. Michel, J. Curély, and A. Dautant, J. Appl. Phys. 76, 754 (1994).

[19] W.T. Thomson, Theory of Vibration with Applications, Chapman \& Hall: London (1996).

[20] G, Meyer and N.M. Amer, Appl. Phys. Lett. 53, 1045 (1988).

[21] C.J. Chen, Scanning Tunneling Microscopy, Oxford University Press New York, NY (1993).

[22] D. Sarid, Scanning Force Microscopy, Oxford University Press, New York, NY (1991).

[23] J.E. Sader and C.P. Green, Rev. Sci. Instrum. 75, 878 (2004).

[24] J.E. Sader, Rev. Sci. Instrum. 74, 2438 (2003). 
This is an author-produced, peer-reviewed version of this article. The final, definitive version of this document can be found online at Review of Scientific Instruments, published by American Institute of Physics. Copyright restrictions may apply. DOI: $10.1063 / 1.3593106$

[25] J.M. Neumeister, Rev. Sci. Instrum. 65, 2527 (1994).

[26] U.D. Schwarz, P. Köster, and R. Wiesendanger, Rev. Sci. Instrum. 67, 2560 (1996).

[27] Bruker Corporation, Probes and Accessories, Bruker, Camarillo, CA (2011).

[28] B. Rogers, L. Manning, T. Sulchek, and J.D. Adams, Ultramicroscopy 100, 267 (2004).

[29] J. Vázquez, M.A. Rivera, J. Hernando, and J.L. Sánchez-Rojas, J. Micromech. Microeng. 19, 015020 (2009).

[30] R. K. Iler, The Chemistry of Silica, Wiley; New York, NY (1979).

[31] H. Choe, M. H. Hong, Y. Seo, K. Lee,G. Kim, Y. Cho, J. Ihm, and W. Jhe, Phys. Rev. Lett. 95, 187801 (2005).

[32] M. Antognozzi, A.D.L. Humphris, and M.J. Miles, Appl. Phys. Lett., 78, 300 (2001).

[33] S. P. Jarvis, T. Uchihashi, T. Ishida, H. Tokumoto, and Y. Nakayama, J. Phys. Chem. B.104, 6091(2000).

[34] S.P. Jarvis, T. Ishida, T. Uchihashi, Y. Nakayama, and H. Tokumoto, Appl. Phys. A. 72, S129 (2001).

[35] S. Jeffery, P. M. Hoffmann, J. B. Pethica, and C. Ramanujan, Phys Rev. B 70, 54114 (2004).

[36] T. Uchihashi, M. Higgins, Y. Nkayama, J. E. Sader, and S. P. Jarvis, Nanotechnology 16, S49. (2005)

[37] M. J. Higgins, M. Polcik, T. Fukuma, J. E. Sader, Yo. Nakayama, and S. P. Jarvis, Biophys J. 91(7), 2532 (2006)

[38] T. D. Li, J. Gao, R. Szoszkiewicz, U. Landman, and E. Riedo, Phys. Rev. B 75, 115415 (2007)

[39] P. M. Chaikin and T. C. Lubensky, Principles of Condensed Matter Physics, Cambridge Press: Cambridge, MA (1995). 
This is an author-produced, peer-reviewed version of this article. The final, definitive version of this document can be found online at Review of Scientific Instruments, published by American Institute of Physics. Copyright restrictions may apply. DOI: $10.1063 / 1.3593106$

\section{Figure Captions}

Figure 1. The optical detection scheme of the AFM using a DMASP tip. The applied modulation and forces also are seen.

Figure 2. (a) Change in $\mathrm{V}_{\mathrm{A}-\mathrm{B}}$ versus cantilever displacement $\left(\mathrm{z}_{\mathrm{c}}\right)$ due to normal force. The slope of the line allows us to determine the conversion factor $\alpha$. (b) The resulting change in $\mathrm{V}_{\mathrm{A}-\mathrm{B}}$ when a voltage is applied to the $\mathrm{ZnO}$ stack. The slope of this line is $\beta$ and is used in the equation to find the voltage-to-force conversion factor.

Figure 3. Force-distance curves for $\mathrm{V}_{\mathrm{A}-\mathrm{B}}(\mathbf{a})$, the frictional force (b), and normal force (c) during the tip-approach towards the surface with velocities of $10 \mathrm{~nm} / \mathrm{s}$ at $55 \%$ relative humidity. The inset in (a) is an expanded plot of $\mathrm{V}_{\mathrm{A}-\mathrm{B}}$ in a range of $8.5-10.5 \mathrm{~nm}$. 
This is an author-produced, peer-reviewed version of this article. The final, definitive version of this document can be found online at Review of Scientific Instruments, published by American Institute of Physics. Copyright restrictions may apply. DOI: $10.1063 / 1.3593106$

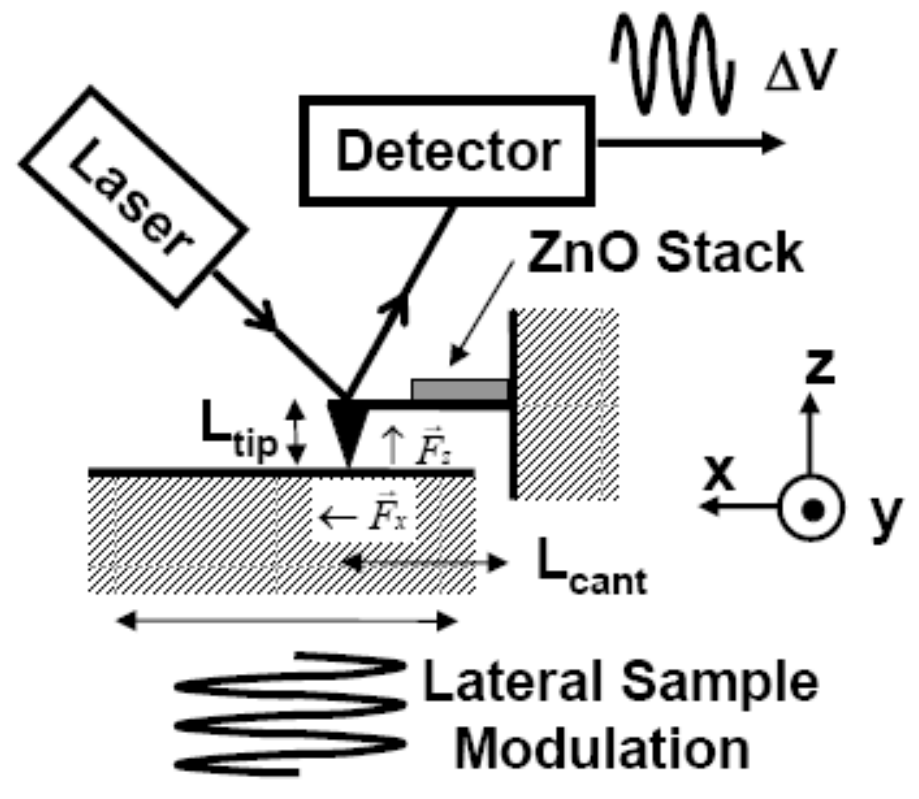

Figure 1 
This is an author-produced, peer-reviewed version of this article. The final, definitive version of this document can be found online at Review of Scientific Instruments, published by American Institute of Physics. Copyright restrictions may apply. DOI: $10.1063 / 1.3593106$
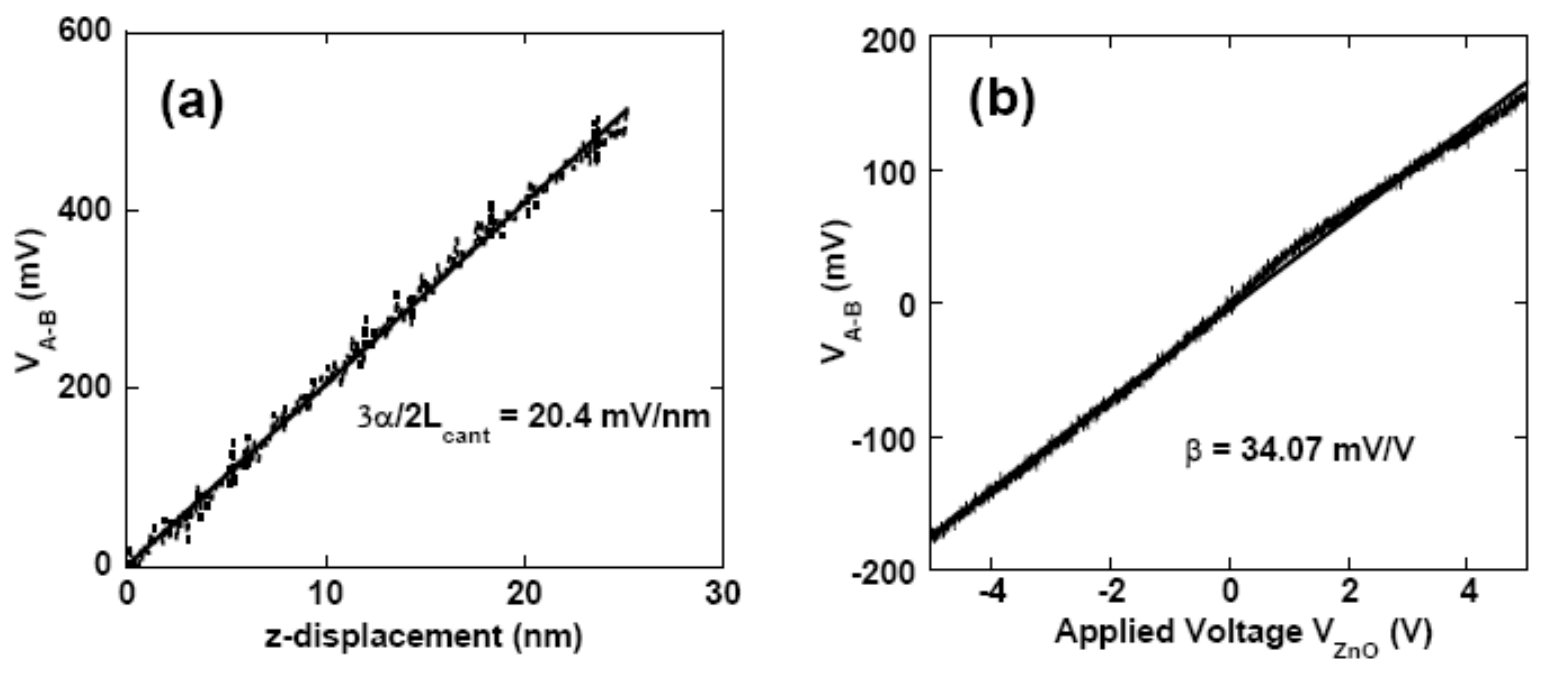

Figure 2 
This is an author-produced, peer-reviewed version of this article. The final, definitive version of this document can be found online at Review of Scientific Instruments, published by American Institute of Physics. Copyright restrictions may apply. DOI: $10.1063 / 1.3593106$
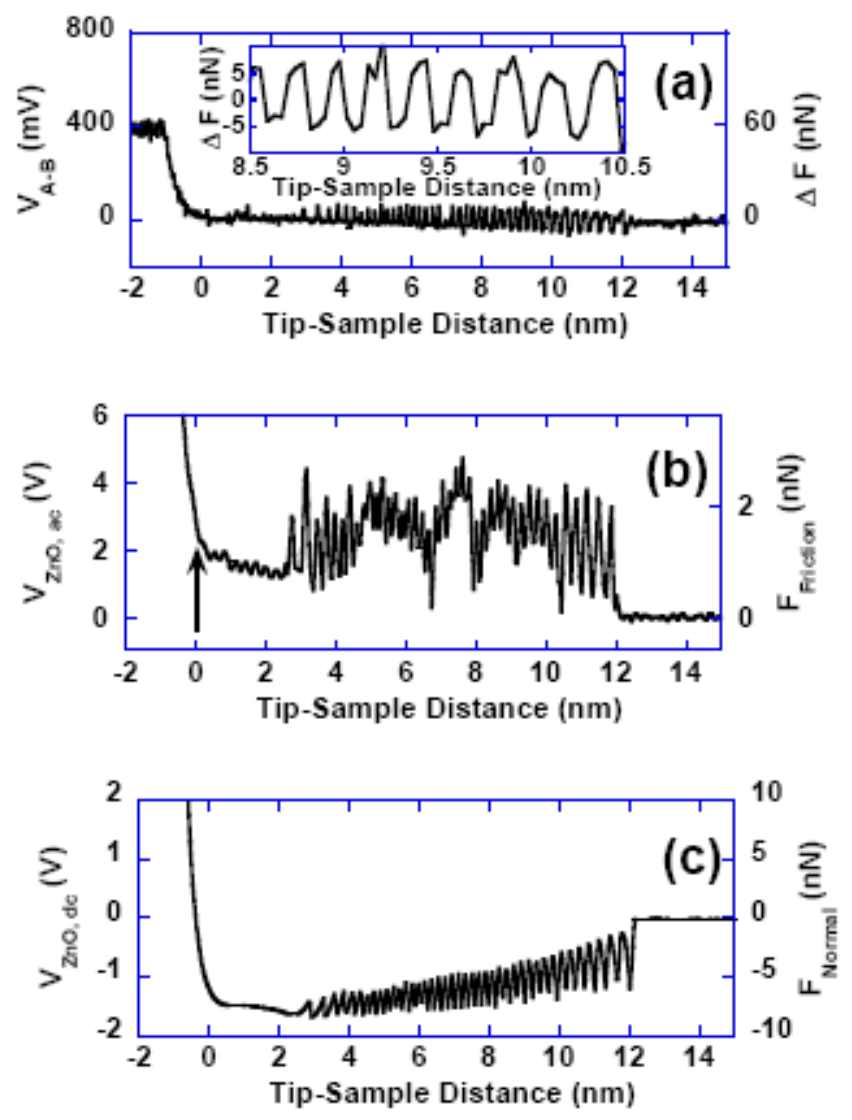

Figure 3 